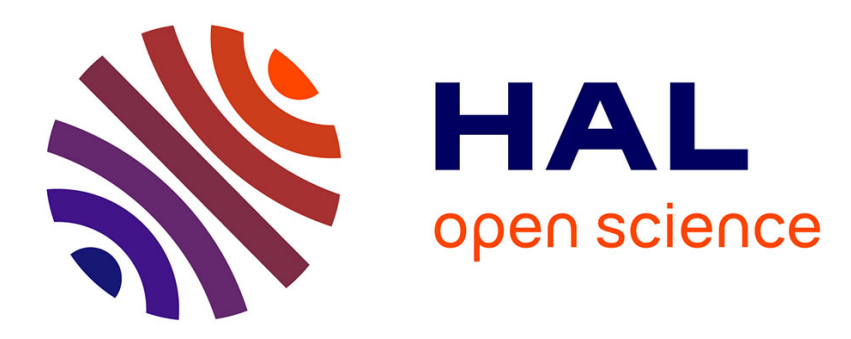

\title{
La reconnaissance de la capacité d'agir sur et par un corps altéré, enjeu de la construction du sujet
}

\author{
Jean-Pierre Garel
}

\section{To cite this version:}

Jean-Pierre Garel. La reconnaissance de la capacité d'agir sur et par un corps altéré, enjeu de la construction du sujet. Eduquer, 2006, "Handicaper" Education corporelle et handicap, 11, pp.61-71. hal-01934082

\section{HAL Id: hal-01934082 \\ https://hal-inshea.archives-ouvertes.fr/hal-01934082}

Submitted on 25 Nov 2018

HAL is a multi-disciplinary open access archive for the deposit and dissemination of scientific research documents, whether they are published or not. The documents may come from teaching and research institutions in France or abroad, or from public or private research centers.
L'archive ouverte pluridisciplinaire HAL, est destinée au dépôt et à la diffusion de documents scientifiques de niveau recherche, publiés ou non, émanant des établissements d'enseignement et de recherche français ou étrangers, des laboratoires publics ou privés. 


\section{Recherches \& éducations}

11 2006 :

Dossier « Handicaper »

Dossier

\section{La reconnaissance de la capacité d'agir sur et par un corps altéré, enjeu de la construction du sujet}

JEAN-PiERre GAREL

\section{Texte intégral}

L'être humain n'accède à un statut de sujet que s'il acquiert, se reconnaît et se voit reconnu une capacité d'agir, à l'opposé de celui qui est assujetti à une puissance extérieure. Cette conception est cohérente avec une approche du sujet « entendu comme tension désirante, pulsionnelle, (...) obstination sauvage à être, à vivre, jouir, pouvoir, posséder, obtenir amour, reconnaissance " (Ardoino, Barus-Michel, 2002, 261), car des capacités sont requises pour que cette tension et ses multiples déclinaisons ne s'épuisent pas totalement en vain.

Envisager le sujet comme capacité et comme être autonome est commun notamment à C. Castoriadis $(1990,191)$ et à V. Descombes, qui écrit que «le concept de sujet dont nous avons besoin est celui de l'agent » et que le " sujet (...) doit être présent dans le monde à la façon d'une puissance causale » (2004, 14-15). Quant à P. Ricœur, évoquant la "capacité de faire arriver des événements dans l'environnement physique et social du sujet agissant ", il voit dans cette puissance d'agir, au sens de l'agency de langue anglaise, une donnée anthropologique fondamentale, c'est-à-dire une caractérisation de l'humain en général $(2004,199)$.

L'humain peut aussi être caractérisé par le besoin de reconnaissance, jugé « vital » par C. Gardou du fait que la reconnaissance « constitue la validité nécessaire de la 
construction de soi » $(1998,99)$. Cette appréciation est partagée par P. Ricœur, qui s'attache plus particulièrement à la reconnaissance des capacités.

L'ensemble de ces considérations conduit à se demander comment la reconnaissance des capacités peut s'effectuer, et ainsi contribuer à la construction de l'individu comme sujet, quand elle porte sur un corps altéré par une déficience motrice précoce, c'est-àdire congénitale ou survenue lors de la première année, et qu'en conséquence le corps échappe au contrôle que le sujet voudrait en avoir pour le mobiliser à travers des habiletés semblables à celles des personnes valides. Est dans ce cas, par exemple, un jeune qui, présentant une infirmité motrice cérébrale (IMC), se heurte à des perturbations du tonus musculaire, de la régulation automatique des mouvements et de leur commande volontaire, ou celui qui, porteur d'une myopathie, est confronté à une diminution progressive de la force contractile des muscles volontaires. Dans quelle mesure le contrôle difficile de son corps par un tel enfant, adolescent ou jeune adulte lui permet-il de se reconnaître et de se voir reconnu la capacité d'agir sur et par son corps ?

\section{Se reconnaître capable d'agir sur et par son corps}

L'enfant IMC dont le corps est sévèrement altéré peut avoir une attitude de négation quant à l'existence de ses incapacités. Conduit à se comparer à des camarades normaux, il est ainsi susceptible de se surestimer. Plus souvent, il est conscient de ses limitations. Selon É Gérard, son sentiment d'impuissance croît avec l'âge, pouvant "éclater violemment » entre 10 et 12 ans (1991).

Les conséquences psychologiques de la déficience sont liées à l'importance des restrictions motrices, même si la singularité des individus n'autorise pas à déduire la manière dont chacun vit et ressent une situation apparemment semblable. Quand l'impotence est considérable, É. Gérard remarque que s'éteignent progressivement les aspirations, l'esprit de compétition et la persévérance, en un mot le désir d' " emprise », entendue comme "conduite coordonnée, développée à plus ou moins long terme en fonction de buts que se propose ou qu'accepte de reprendre en charge la personne qui s'y engage » (Perron, cité par Gérard, ibid., 188). Le sentiment d'impuissance tient notamment aux échecs répétés dans les tentatives de maîtriser l'environnement et aux inévitables dépendances. Dans le cas d'une déficience motrice évolutive, une myopathie de Duchenne par exemple, la perte de la marche et de la force musculaire risque d'induire " une inhibition parfois massive, fantasmatique et/ou relationnelle, le désinvestissement du monde extérieur ou scolaire et la dépression »(Benony, 1986).

Lorsque les enfants qui présentent une déficience motrice parviennent à l'adolescence, c'est-à-dire à cette période de quête identitaire caractérisée notamment par une revendication d'indépendance dans tous les domaines, une faible autonomie corporelle augmente la prévalence des problèmes psychologiques ou des difficultés d'adaptation (Garel, 2005).

$8 \quad$ Faute d'une maîtrise gestuelle suffisante, certains enfants doivent s'en remettre à un tiers pour les soins du corps et l'habillement. Au moment de l'adolescence, l'intrusion dans l'intimité corporelle peut être mal vécue, de même que l'impossibilité d'assumer seul des actes qui relèvent d'un souci de soi légitime. Cloé, 13 ans, qui souffre d'arthrite chronique juvénile, confie : «Pour me coiffer, j’ai besoin de quelqu'un, je peux pas me coiffer toute seule. Je peux pas avec mes mains, j’y arrive pas. J'aime bien la queue de cheval haute, mais basse ou haute, j'ai essayé plusieurs fois, ça marche pas ». 
L'ensemble des obstacles rencontrés dans les tentatives de mobilisation corporelle contribue à l'émergence d'une identité négative. Posant à des enfants IMC la question «peux-tu me dire qui tu es ?", É. Gérard note la fréquence de la réponse : «je suis un handicapé » (ibid.). Cependant, les atteintes motrices et l'intériorisation d'une image de soi comme incapable n'empêchent pas fatalement les enfants et les adolescents IMC d'exprimer leur désir d'une mise en jeu corporelle dans des activités ludiques ou sportives. Dans une recherche analysant les récits spontanés qu'ils formulent, É. Gérard note une " inflation prodigieuse » du thème « jeux et sports » chez les adolescents (ibid.). De son côté, F. de Barbot remarque que des enfants IMC présentant une déficience motrice très invalidante, accueillis dans le service de rééducation neurologique infantile de l'hôpital de Bicêtre, manifestent une véritable « jubilation » dans des jeux moteurs symboliques où ils sont chevaux " galopants ", " acrobates ", etc. (1987). Lorsque les adolescents de ce service réclament de faire du sport, F. de Barbot regrette que cette demande soit trop souvent entendue par les adultes comme un refus, une négation du handicap. "Ne devrait-on pas y reconnaître d'abord, se demande-t-elle, la recherche d'un plaisir rarement offert, rarement atteint, souvent contrecarré par les exigences de la rééducation et la prévention orthopédique? » (Ibid., 221).

Le plaisir d'une mise en jeu corporelle n'est pas recherché uniquement dans les activités sportives. Les activités physiques artistiques, la danse par exemple, suscitent un intérêt particulier malgré des troubles moteurs importants et la distance par rapport aux normes esthétiques socialement valorisées (Garel, 2002). Ce qu'y trouvent des personnes en situation de handicap moteur semble tenir notamment à la liberté qu'offrent ces activités, à l'opposé des contraintes thérapeutiques et rééducatives dont les visées compensatrices ne laissent pas toujours une place suffisante à l'expression personnelle. Florence, une jeune adulte IMC, témoigne après un stage de danse : "Sortir du fauteuil, m'exprimer, ça c'est important, m'exprimer en mouvement ; par terre je me sens libre ». Et puis il y a la gratification de découvrir ses capacités : «Je trouve que je ne profite pas assez de mes capacités (...) Je n'arrivais pas à croire que j'étais capable de faire tant de choses avec mon corps ». Ce changement de regard contribue à une évolution de l'identité que s'attribue le sujet : "Maintenant quand je danse, je suis quelqu'un d'autre ", déclare un adolescent IMC au retour d'un festival de danse.

L'action sur le corps a aussi pour cadre les rééducations physiques. Elles peuvent être subies, ou bien être l'objet d'un investissement actif de la part du jeune. C'est le cas de Camille, 10 ans, consciente de son état physique induit par une lésion cérébrale, de ses progrès, du travail qui lui reste à accomplir et dans lequel elle se situe comme sujet : "Même si mon corps va bien, je marche et tout ça, j'ai encore des problèmes (...) II faut que j'essaie de remettre ma colonne droite et que j'allonge un peu ma jambe ».

Le souci de demeurer acteur dans les activités de la vie quotidienne concerne notamment les soins du corps. Si elle est devenue peu à peu dépendante d'une tierce personne, Cloé s'efforce de sauvegarder la plus grande autonomie possible : "Pour faire ma toilette, avant j'avais besoin de personne, maintenant j'ai besoin de quelqu'un. Donc, je fais tout ce que je peux faire toute seule, et quand j'ai besoin d'aide j'appelle quelqu'un pour m'aider. Moi, je peux mettre le haut par exemple, je peux faire des choses ».

Pour un jeune dont les possibilités physiques sont limitées, la reconnaissance de ses capacités d'agir sur et par son corps doit s'accompagner de celle de ses limites, afin de pouvoir recourir si besoin à un tiers dès qu'une tâche devient trop exigeante. Mais le rôle principal d'autrui par rapport à la reconnaissance n'est pas là. Il est avant tout à considérer au niveau de son enjeu et de son processus : pourquoi et comment les autres 


\section{Se voir reconnaître la capacité d'agir}

À propos des personnes en situation de handicap, C. Gardou écrit qu' « il n'est pas de sujet sans un autre qui le reconnaisse comme tel dans sa différence » (ibid.). Assertion à laquelle nous ajouterons « et dans sa similitude », parce que l'altérité n'est jamais si radicale qu'elle exclut toute ressemblance. Faute de faire droit à ce qui réunit comme à ce qui distingue, on risque de répondre à la demande de reconnaissance par une politique différentialiste aboutissant à des replis identitaires.

Plus ou moins différent, celui qui est « handicapé » n'échappe pas à l'affirmation selon laquelle "la reconnaissance de soi comme homme "capable" de certains accomplissements (...) requiert l'aide d'autrui » (Ricœur, ibid., 109). Le sujet dont on envisage la construction ne se conçoit donc pas sans les autres. Il est social, à l'inverse de l'individu, création de la modernité et rêve d'une indépendance qui conduit à un « individualisme désocialisant » (Renaut, 1989, 92). Cette conception intersubjective peut donner lieu à une approche de la reconnaissance selon trois modèles différents, transposés de ceux qu'A. Honneth a empruntés à Hegel dans sa réflexion sur la lutte pour la reconnaissance (2000).

Le premier modèle de la reconnaissance intersubjective retenu par Honneth est placé sous l'égide de l'amour, c'est-à-dire des « liens affectifs puissants entre un petit nombre de personnes » (ibid., 117). En le transposant dans une perspective interactionniste, on retiendra que l'image de soi, composante fondamentale de la construction identitaire, est sensible à l'image attribuée et renvoyée par autrui, notamment par les « autrui significatifs » (Mead, 1963), en d'autres termes ceux qui comptent aux yeux du jeune : parents, enseignants, camarades... De ce point de vue, se pose donc la question de l'image qui résulte de l'interaction avec ces proches.

É. Gérard envisage les conséquences du décalage pouvant exister entre l'enfant désiré par les parents et l'enfant réel, en l'occurrence IMC. La conscience d'être éloigné de ce désir est susceptible d'avoir sur cet enfant un effet culpabilisant, alimenté par un sentiment d'impuissance, parce qu'il ne peut pas faire mieux. Dès lors, il lui est difficile d'investir un corps plus ou moins refusé par les proches, d'avoir une confiance en soi suffisante pour oser s'engager dans des activités physiques, d'autant plus qu'ils ne croient guère en ses possibilités et craignent un accident qui accentuerait les désordres physiques. Il risque alors de ne pas être suffisamment stimulé et étayé dans ses tentatives de mise en jeu corporelle et d'emprise sur son environnement. "Comment, demande É. Gérard, peut-il évoluer, se construire et s'affirmer une personnalité s'il vit près de personnes qui craignent tout pour lui et ne l'autorisent à aucune expérience personnelle (...) s'il est sans espoir d'être épaulé ou encouragé lors d'une tentative personnelle?» (Ibid., 180).

Dans le domaine scolaire, le rôle de la confiance accordée par les enseignants aux élèves en situation de handicap mérite d'être souligné. Julie, collégienne IMC, questionnée sur ce qui lui paraît fondamental dans sa réussite scolaire, évoque cet aspect en premier : "Quand on a des problèmes, on peut leur (les professeurs) en parler (...) ils nous encouragent, ils nous disent " mais si, tu vas y arriver ", et on se rend compte qu'on y arrive. Ils ont confiance en nous ».

Que les autres doutent de leurs capacités peut constituer un défi stimulant pour des personnes " handicapées ", tel Joël, adolescent IMC : "J'aime bien danser pour prouver que nous sommes capables de faire des choses comme les personnes qui ne 
sont pas handicapées ». Mais les regards extérieurs constituent pour certains une épreuve redoutable, comme ce fut le cas pour Céline, jeune adulte IMC, lors d'un stage de danse : "Je me suis mise à pleurer. J'avais une angoisse. Il fallait que je montre mon travail et pour moi c'était dur de le montrer, que les autres regardent. Je ne me sentais pas à l'aise. J'avais peur de ne pas réussir ».

Le second modèle de la reconnaissance, selon Honneth, est de nature juridique. Il s'agit de reconnaître le droit, pour tous, d'acquérir des capacités personnelles, ce qui suppose, notamment, que l'école s'attache simultanément à l'égalité des droits et à l'équité de traitement des élèves en fonction des différences individuelles. Par ailleurs, il ne suffit pas d'avoir acquis des capacités reconnues par soi-même et par les autres si les activités dans lesquelles on pourrait et souhaiterait les exercer sont inaccessibles (physiquement, financièrement, ou faute d'une structure sportive ouverte aux personnes « handicapées »). La reconnaissance du droit d'accès aux biens et services destinés à tous doit donc comprendre des droits qui garantissent cet accès.

Le troisième $\mathrm{m}$ odèle se réfère à l'estime sociale. Ici, les relations interpersonnelles sont travaillées par une culture commune, par des pratiques sociales dont un enjeu est " l'instauration du lien social et des modalités d'identité qui s'y rattachent » (Ricœur, ibid., 202). Ces pratiques forgent des représentations partagées, une reconnaissance mutuelle qui fonde le « je » sur le « nous ». Le couplage entre représentations collectives et pratiques sociales justifie l'idée de capacités sociales (Ricœur, ibid., 203) et l'estime sociale auxquelles elles peuvent donner lieu.

Pour favoriser la reconnaissance des capacités sociales dans le domaine des pratiques corporelles, le choix des activités se porte logiquement sur des pratiques sociales valorisées et valorisantes. Sont donc essentiellement retenues les activités physiques de nature sportive et artistique, plutôt que celles qui relèvent d'une approche thérapeutique et qui se focalisent sur la réadaptation.

L' « estime sociale » est également privilégiée par l'« externalisation 》 des productions (Bruner, 1996, 39), en l'occurrence corporelles. L'externalisation, c'est la production d' « œuvres », qui est, selon I. Meyerson, la fonction principale de toute activité culturelle collective (1948). Dans leur version mineure (à côté des arts, de la culture, des institutions...), les œuvres sont « celles de petits groupes, qui procurent à la fois fierté, identité et sens de la continuité à ceux qui, aussi peu que ce soit, ont participé à leur élaboration » (Meyerson, cité par Bruner, ibid.). L'enjeu en est défini par C. Gardou : "Le sujet ne se déploie que si nous lui permettons de réaliser son œuvre, fût-elle immensément modeste ou insignifiante à nos yeux. Car l'homme chacun l'expérimente - n'existe vraiment qu'à travers ses oeuvres : ses gestes, sa parole, ses actes » (ibid., 100).

Dans cette perspective, produire une œuvre par son activité physique c'est donner à voir ses résultats, et si possible en laisser une trace. Les activités à caractère artistique sont un bon exemple, mais non exclusif. Toute activité peut être montrée aux autres selon des modalités diverses (dans l'instant, ou en différé par une photo, voire un film); l'évaluation, en EPS, peut être consignée sur une fiche ou un cahier ; les rencontres sportives peuvent donner lieu à une remise de médailles, de coupes, de diplômes...

L'estime sociale qui peut être accordée à des personnes au corps altéré, au vu de leurs prestations physiques, ne va pas de soi. En effet, dans une société qui met en avant le culte de la performance et la référence à un corps beau et sain, la faiblesse et la distance par rapport aux canons esthétiques en vigueur sont a priori l'objet d'une stigmatisation plus ou moins prononcée. Après bien d'autres, M. M. Marzano-Parisoli défend cette thèse, mais formule une remarque qui conduit à la relativiser. En l'occurrence, elle souligne la valeur sociale actuellement accordée au contrôle de soi : "Si le contrôle de soi exprime, en effet, la valeur la plus positive, l'absence de contrôle exprime, au 
contraire, l'impuissance de ceux qui, ne pouvant exhiber un corps musclé/mince, sont censés être incapables d'assurer un contrôle sur leur vie et sur leurs instincts les plus primitifs » $(2002,19)$.

Dans ces conditions, ce sont donc moins les caractéristiques et les qualités physiques qui priment que « les efforts accomplis par les individus afin de modifier leur corps et acquérir ainsi par la volonté les caractéristiques requises par la société » (ibid., p. 22). Or l'enfant ou l'adolescent porteur d'une déficience motrice, par ce que l'EPS constitue pour lui un enjeu très fort, témoigne souvent dans cette discipline d'une motivation et d'une persévérance qui constituent un modèle pour les autres élèves. Des professeurs d'EPS soulignent cet effet stimulant sur le groupe classe. Si on considère également les résultats souvent obtenus par ces jeunes dans cette discipline, qui surprennent leurs professeurs et leurs camarades, on en arrive à remettre en question l'idée qu'un jeune présentant une déficience motrice est fatalement stigmatisé par le regard des autres quand son corps est en scène en milieu ordinaire. Mais la pertinence des efforts physiques accomplis n'est plus assurée dès lors que le corps est tellement investi qu'il est l'objet d'un travail obsessionnel et d'un narcissisme aliénant.

\section{Conclusion}

Il est apparu difficile, pour un jeune dont le corps est altéré par une déficience motrice précoce, de se reconnaitre capable de mobiliser son corps. Pourtant, les obstacles susceptibles d'entraver cette reconnaissance et d'instiller un sentiment d'impuissance ne sont pas tels qu'ils soient fatalement infranchissables. Même dans le cas de déficiences très invalidantes, des exemples montrent le maintien du désir de mettre en jeu son corps et des mises en jeu effectives, révélatrices de potentialités parfois insoupçonnées, dans la pratique d'activités physiques, sportives et artistiques comme dans les activités de la vie quotidienne. À travers l'activité qu'il y déploie, l'individu peut alors se reconnaître et se voir reconnu la capacité d'agir sur et par son corps, ainsi que s'y reconnaître, c'est-à-dire se construire comme sujet tendant vers l'autonomie et se donner une identité. Cette activité est d'autant plus féconde que celui qui s'y engage est accompagné, autant que de besoin, par des professionnels compétents et que les significations qu'il lui accorde lui permettent de nourrir des projets personnels, d'en réaliser, et d'instaurer du lien social.

\section{Bibliographie}

Ardoino J., Barus-Michel J. (2002). "Sujet », in J. Barus-Michel, E. Enriquez, A. Lévy, Vocabulaire de psychosociologie, Toulouse, Érès, p. 258-265.

Barbot de F. (1987). "Enfant handicapé moteur : enfant malade ? ", in P. Ferrari et R. Lazarovici, (dir.), L'enfant malade et son corps, Toulouse, Privat.

Benony H. (1986). « Approche psychopathologique des adolescents myopathes », Perspectives psychiatriques, $\mathrm{n}^{\circ}$ 2, $\mathrm{p} .110-124$.

Bruner J. (1996). L'éducation, entrée dans la culture. Les problèmes de l'école à la lumière de la psychologie culturelle, Paris, Retz.

Castoriadis C. (1990). Le monde morcelé, Paris, Seuil.

Descombes V. (2004) Le complément de sujet. Enquête sur le fait d'agir de soi-même, Paris, Gallimard.

Gardou C. (1998). « La personne handicapée : d'objet à sujet, de l'intention à l'acte », Colloque, Lyon II, 17-19 sept 1998, La Nouvelle revue de l'AIS, Éd. du Cnefei, nº 4, p. 97-109. 
Garel J-P. (2002). « Expressions corporelles à l'épreuve d'altérations du corps et des sens », $L a$ Nouvelle revue de l'AIS, Éd. du Cnefei, $\mathrm{n}^{\circ}{ }^{18}$, p. 97-106.

Garel J-P. (2005). Adolescents au corps altéré et apprentissages par corps, La Nouvelle Revue de l'AIS, Éd. du Cnefei, nº 29 , p. 33-46.

Gérard É. (1991). « Être infirme moteur cérébral », in R. Perron (dir.), Les représentations de soi : développements, dynamiques, conflits, Toulouse, Privat.

Honneth A. (2000). La lutte pour la reconnaissance. Grammaire morale des conflits sociaux, Paris, Éd. du Cerf, coll. Passages.

Marzano-Parasoli M. M. (2002). Penser le corps, Paris, PUF.

Mead G. H. (1963). L'esprit, le soi et la société, Paris, PUF.

Meyerson I. (1995). Les fonctions psychologiques des œuvres, Paris, Albin Michel.

Renaut A. (1989). L’ère de l'individu, Paris, Gallimard.

Ricœur P. (2004). Parcours de la reconnaissance, Paris, Stock.

\section{Pour citer cet article}

Référence électronique

Jean-Pierre Garel, « La reconnaissance de la capacité d'agir sur et par un corps altéré, enjeu de la construction du sujet », Éduquer [En ligne], 11 | 2006, mis en ligne le 15 octobre 2008, consulté le 24 novembre 2018. URL :

http://journals.openedition.org/rechercheseducations/388

\section{Auteur}

Jean-Pierre Garel

Laboratoire Relacs (Université du Littoral Côte d'Opale)

\section{Droits d'auteur}

Propriété intellectuelle 